\title{
Recomendaciones para la ejecución de anestesia regional no obstétrica en perioperatorio de pacientes COVID-19
}

Grupo de trabajo. Comité de Anestesia Regional de la Sociedad de Anestesiología de Chile. LASRA Chile. Aliste, Julián; Altermatt, Fernando; Atton, Rousmary; Bravo, Daniela; Layera, Sebastián; Miranda, Pablo; Pesce, Ítalo.

\section{Desarrollo de la Recomendación Clínica (RC)}

\subsection{Introducción}

n diciembre de 2019, surgió una serie de casos de neumonía causada por un nuevo coronavirus, denominado 2019-nCoV o SARS-CoV2. La propagación del virus ha sido extremadamente rápida y la organización mundial de la salud declaró a la enfermedad COVID-19, causada por 2019-nCoV, como una pandemia. En este contexto la Sociedad de Anestesiología de Chile (SACH) ha elaborado recomendaciones generales para el manejo perioperatorio de los pacientes sospechosos y/o portadores de la enfermedad. Sin embargo, dado que durante el levantamiento de evidencia para la confección de dicha guía se objetivó la ausencia de sugerencias específicas para la ejecución de técnicas de anestesia regional fuera del ambiente obstétrico, se solicitó desde el Comité Científico de SACH al Comité de Anestesia Regional de SACH (CARSACH), representante LASRA (LatinAmerican Society of Regional Anesthesia) en Chile, a través de un grupo de expertos, confeccionar recomendaciones locales en esta materia.

Estas recomendaciones vienen a complementar las normativas generales SACH y las impuestas por las autoridades sanitarias del país en un aspecto particular como es el período perioperatorio. La SACH y CARSACH-LASRA Chile esperan que estas recomendaciones contribuyan a establecer medidas eficaces para disminuir la propagación del COVID-19 y puedan servir de guía a las autoridades pertinentes y anestesiólogos en el manejo perioperatorio de "casos" COVID-19. Como la definición de "caso" son dinámicas se sugiere remitir a la página del Ministerio de Salud confirmar periódicamente al respecto (ver referencias). Además, dada la continua aparición de nuevas evidencias respecto a esta patología, a partir de su publicación, estas recomendaciones estarán en constante revisión por el mismo comité que las creó, para evaluar eventuales modificaciones o actualizaciones.

\subsection{Objetivo general}

Recomendar cómo manejar el perioperatorio de un individuo con sospecha o confirmación de infección por COVID-19 donde se ejecutará una técnica de anestesia regional, anestésica o analgésica, disminuyendo el riesgo de infección del personal de salud y otros sujetos hospitalizados, aunque manteniendo los estándares de seguridad para el paciente.

\subsection{Objetivos específicos}

- Recomendar las medidas de preparación necesarias previo a la ejecución de técnicas de anestesia regional durante el manejo perioperatorio de un paciente COVID-19.

- Recomendar cómo ejecutar bloqueos regionales, neuroaxiales o periféricos, tanto anestésicos y/o analgésicos en pacientes COVID-19, así como el manejo intraoperatorio de estos.

- Recomendar conductas respecto al manejo postoperatorio de pacientes COVID-19 que han recibido bloqueos regionales.

\subsection{Consideraciones}

El manejo de pacientes con diagnóstico confirmado o sospecha de infección por COVID-19 representa un riesgo considerable de contagio para todo el personal de salud (PS). El tránsito perioperatorio del paciente implica una serie de procedimientos que aumentan el riesgo de contagio al PS, especialmente cuando se debe manejar avanzadamente la vía aérea (intubación/extubación). 
Es importante enfatizar que la anestesia general no está particularmente contraindicada en casos COVID-19, sin embargo el manejo obligado de la vía aérea que ésta conlleva la transforma en un riesgo para el PS involucrado en su administración. En este contexto, con el fin de disminuir la generación de aerosoles, la anestesia regional neuroaxial y/o periférica representa una alternativa a la anestesia general; siempre y cuando el operador tenga la experiencia suficiente para asegurar una eficacia razonable y la patología quirúrgica del paciente sea susceptible de resolverse a través de la técnica anestésica seleccionada, de manera tal de permitir condiciones óptimas, tanto quirúrgicas como de seguridad para el paciente y el PS.

Es importante también considerar que en un paciente altamente sintomático respiratorio (tos o disnea con altos requerimientos de oxígeno suplementario), la anestesia regional no es sinónimo de ausencia de producción de aerosoles y una criteriosa evaluación caso a caso es siempre necesaria, considerando incluso que la conducta puede cambiar durante la evolución de un caso en particular.

Un comité conformado por siete expertos en anestesia regional de distintos centros universitarios con programas de perfeccionamiento (Fellowship) en anestesia regional en nuestro país y miembros de CARSACH-LASRA Chile, participó en la confección de las presentes recomendaciones respecto a la ejecución de bloqueos regionales (neuroaxiales o periféricos) anestésicos o analgésicos en casos COVID-19.

\section{Aspectos generales}

1. PREMISAS para ANESTESIA REGIONAL en paciente COVID-19:
a. Disminuir al máximo el riesgo de aerosoli- zación del virus.
b. Asegurar por sobre todo la seguridad del paciente y del PS.
c. Elegir una técnica anestésica con baja tasa de falla que brinde adecuadas condicio- nes quirúrgicas.
d. Realizar la técnica que mejor se domine con las herramientas que cuente en el centro de trabajo.

2. El control de la vía aérea en pacientes COVID-19 ha sido bien normado por diversas sociedades a nivel internacional, y por recomendaciones locales, generadas por la Sociedad de Anestesiología de Chile (SACH). Un manejo adecuado de la vía aérea de estos pacientes debe seguir las recomendaciones vigentes.
3. De ser necesario una anestesia general bajo los términos previamente descritos, ésta puede ser complementada con bloqueos regionales, neuroaxiales o periféricos, de no estar contraindicados y estimarse de beneficio para el manejo perioperatorio del paciente.

a. En este escenario, dado el menor riesgo teórico de contaminación, es posible ejecutar las técnicas regionales periféricas con el paciente anestesiado y con la vía aérea asegurada, si el operador es un experto, y se disponen y siguen todas las medidas que permitan disminuir al máximo el riesgo daño neurológico.

b. En el caso de un bloqueo neuroaxial complementario a anestesia general, nuestra primera opción será realizarlo con el paciente despierto y cooperativo, idealmente en decúbito lateral.

4. La ejecución de bloqueos analgésicos en dosis única (con o sin coadyuvantes) o continuos, puede disminuir o evitar el requerimiento de analgesia de rescate. No existe contraindicación formal a esquemas de analgesia multimodal.

a. Una adecuada analgesia regional permitiría disminuir la necesidad de intervenciones con exposición del PS en el post operatorio, principalmente al utilizar modalidades controladas por paciente (PCA).

b. Una adecuada analgesia regional permitiría ahorrar en uso de opioides disminuyendo el riesgo de náuseas y vómitos postoperatorios (NVPO), y secundariamente la posibilidad de contaminación asociada en casos COVID-19.

c. Una adecuada analgesia regional permitiría ahorrar AINES, que dado su incierto rol agravante en la evolución de estos pacientes, representaría otro beneficio teórico adicional en casos COVID-19.

d. El uso de un bloqueo regional anestésico representa una alternativa para disminuir la exposición a aerosoles si las condiciones respiratorias basales del paciente son buenas y éstas se mantengan sin cambios durante la cirugía. Sin embargo, todo el PS debe utilizar el equipo de protección personal (EPP) recomendado para el manejo de casos COVID-19.

5. Es altamente recomendable una estrecha comunicación con equipo tratante, tanto médico como quirúrgico, para consensuar el manejo perioperatorio. 


\section{Recomendaciones}

\subsection{Preparación}

1. Se deben respetar las recomendaciones de parámetros de laboratorio y tiempos de suspensión/ reinicio de terapias anticoagulantes. Excepciones deben evaluarse caso a caso según riesgo/ beneficio [Recomendaciones y Guías ASRA]. No se recomienda solicitar exámenes de coagulación de rutina y se debe correlacionar con la clínica.

2. Todo caso COVID-19 que se prevé que será operado con una técnica regional debe ser trasladado directamente al pabellón correspondiente para el manejo de estos pacientes según recomendaciones preestablecidas, y así realizar el bloqueo dentro de pabellón.

3. La evaluación preanestésica, definición del plan anestésico y obtención del consentimiento informado debe realizarse donde lo estipule cada institución. Sin embargo, debiera privilegiarse disminuir al mínimo el número de exposiciones y contactos con el paciente fuera del pabellón.

a. El plan anestésico se puede explicar y discutir en el mismo lugar dando tiempo al paciente para que lo entienda y resuelva sus dudas.

b. Debiera evitarse la firma de documentos; favoreciendo la autorización verbal de procedimientos, idealmente con testigos y registro en ficha clínica.

c. En caso de que la firma del consentimiento sea institucionalmente inevitable, una alternativa es que lo haga un representante no contagiado.

4. El pabellón predeterminado para el manejo de estos pacientes deberá disponer de todo lo necesario para la ejecución tanto de anestesia general como anestesia regional según el estándar institucional para un pabellón quirúrgico.

5. Tanto técnicas de bloqueo neuroaxial como periférico deberán ser realizadas en pabeIlón, en pacientes debidamente monitorizados y teniendo acceso a todas las medidas estándar recomendadas. Deben estar disponibles todos los fármacos e insumos necesarios previo a la llegada del paciente al pabellón.

6. Se debe disponer de los insumos y equipos de reanimación necesarios ante un episodio de intoxi- cación por anestésico local. Estos insumos deben encontrarse inmediatamente disponibles, pero no en el pabellón, de forma de evitar el tener que desecharlos innecesariamente.

7. Se deberá disponer siempre de todo el material necesario para seguir la recomendación para inducción de anestesia general y control de vía aérea acorde a paciente COVID-19. Todo paciente que va a ser sometido a una técnica regional como anestesia única tiene el riesgo potencial de requerir una conversión a anestesia general. Deben tomarse por tanto todas las medidas requeridas para disponer del material y entrenamiento necesarios para el manejo de una vía aérea según las recomendaciones generadas para tal efecto. El método de elección de manejo la vía aérea es la intubación orotraqueal, ejecutada según las recomendaciones vigentes. El uso de dispositivos supraglóticos son sólo recomendadas como plan alternativo ante una vía aérea difícil.

8. Los procedimientos regionales deben ser realizados por el profesional más experimentado, con el objetivo de disminuir el riesgo de falla de la técnica al mínimo.

9. El uso de EPP es obligatorio en caso de tener contacto directo con pacientes COVID-19. Aunque la anestesia regional no está considerada como un procedimiento generador de aerosoles, en condiciones óptimas de recursos, se sugiere estar preparado ante un eventual manejo de la vía aérea con:

a. Protección ocular con antiparras o escudo facial.

b. Mascarilla N95.

c. Bata quirúrgica desechable.

d. Doble guante (segundo par debe ser estéril instalado para realización del procedimiento).

10. Si los pacientes COVID-19 reciben anestesia regional, sólo es necesario que utilicen mascarilla quirúrgica durante el procedimiento. De ser posible, se sugiere además utilizar una sábana plástica transparente para aislar la cabeza del paciente.

11. Si se decide realizar un bloqueo regional guiado por ecografía, se recomienda cubrir el equipo completamente con un plástico transparen- 


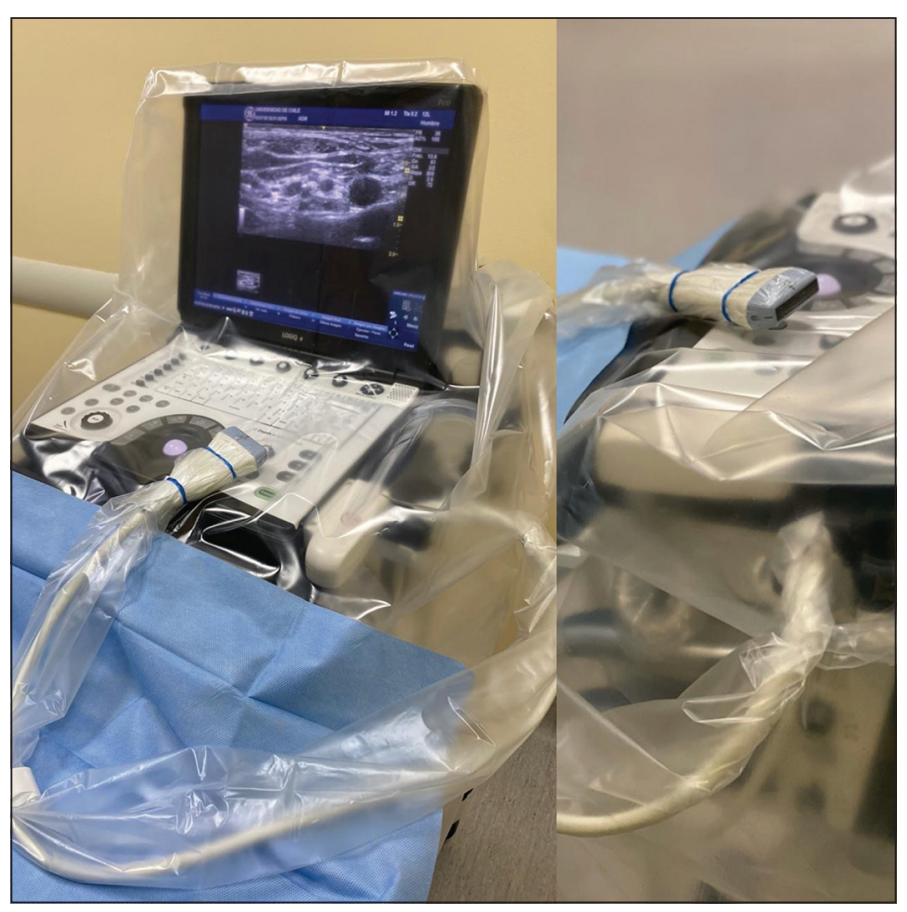

Figura 1. Protección de maquina de ultrasonido, tanto equipo como transductor deben ser cubiertos con plástico. te y el uso de una manga estéril larga para minimizar el contacto directo del transductor con el paciente (figura 1). Si no dispone de ella, utilice una manga de laparoscopía.

\subsection{Procedimiento e intraoperatorio}

1. Se desaconseja la administración de oxígeno suplementario en alto flujo (ej. mascarilla Venturi, cánula nasal de alto flujo, CPAP, etc.). Si el paciente lo requiere, se sugiere el uso de naricera bajo la mascarilla quirúrgica con flujos bajos.

2. Si se decide utilizar un bloqueo regional como técnica anestésica, es recomendable que el paciente pueda mantener su capacidad ventilatoria inalterada durante la cirugía y postoperatorio, al igual que un estado de conciencia apto para obedecer órdenes y mantener autocontrol.

3. Se recomienda evitar nivel de sedación intravenosa que condicione el uso de oxigenoterapia durante el procedimiento.

4. En caso de procedimientos con anestesia neuroaxial como técnica preferente, el uso de técnicas combinadas (espinal-peridural) puede otor- gar mayor flexibilidad ante una duración variable del procedimiento. Esta técnica permitiría disminuir las dosis de anestésicos local y la duración bloqueo motor postoperatorio, lo que permitiría un alta acelerada desde el pabellón hacia su unidad de origen.

5. Se debe tener especial precaución con los bloqueos de extremidad superior en pacientes sintomáticos respiratorios por el riesgo de parálisis diafragmática y empeoramiento de su función pulmonar.

6. Siempre se deben respetar las dosis máximas de anestésicos locales recomendadas.

\subsection{Postoperatorio}

1. Analgesia postoperatoria

a. El uso de AINES ha sido controversial en pacientes con COVID19. No existe evidencia robusta que lo contraindique. Dado que tampoco se sabe el efecto que tiene una analgesia deficiente en el curso de la evolución de pacientes con esta patología, recomendaríamos mantener su uso como parte de un esquema multimodal. 
b. El uso de opioides puede asociarse a aumento del riesgo de NVPO. Se recomienda el uso de técnicas que disminuyan este riesgo, como la analgesia regional, y el uso de profilaxis antiemética.

c. El uso de técnicas continuas, permite entregar una analgesia de excelente calidad en el período postoperatorio. De contarse con sistemas de infusión continuos o de analgesia controlada por el paciente, permite además disminuir la interacción del personal de salud con el paciente, evitando la administración repetida de analgesia de rescate por métodos distintos a los ya instalados (administración intravenosa).

d. En bloqueos continuos de nervio periférico (BCNP), propiciar el uso de sistemas desechables de infusión de estar disponibles.

e. En BCNP, utilizar soluciones con concentraciones bajas de anestésico local que favorezcan bloqueo sensitivo y no motor para optimizar analgesia y rehabilitación.

f. Ante la imposibilidad de utilizar técnicas continuas periféricas, no hay contraindicación formal al uso de coadyuvantes para los bloqueos en estos pacientes.
2. Una vez que las condiciones del paciente lo permitan, de acuerdo a las escalas de valoración de cada institución, el traslado a su unidad de destino será en forma directa desde el pabeIlón, evitando su traslado a URPA.

3. De ser posible, en pacientes COVID-19 las visitas de servicios de dolor agudo deberán programarse con posterioridad a la de los pacientes NO COVID-19. Coordinarse con el equipo de planta a cargo de estos pacientes, para evitar contactos innecesarios.

4. De ser imprescindible, el contacto en la visita de pacientes COVID-19 por el servicio de dolor agudo deberá seguir las recomendaciones de EPP del servicio de hospitalización del paciente.

5. No existe contraindicación al uso de analgesia regional domiciliaria en casos COVID-19, si ésta es una práctica institucional habitual y se estima conveniente. Debe privilegiarse el control telefónico estricto y auto-retiro de catéteres, evitando que el paciente concurra innecesariamente a control.

\section{Referencias}

1. Aranda F, Aliste J, Altermatt F, Alvarez JP, Bernucci F, Cabrera MC, Carrasco E, De la Fuente R, Egaña Jl, Lacassie H, Merino W, Penna A, Torres D. Recomendaciones para el manejo de pacientes con COVID19 en el perioperatorio. Revista Chilena de Anestesia. Asociacion de Medicos Anestesiologos de Chile; 2020 Mar 31;49(2):196-202. http://dx.doi.org/10.25237/revchilanestv49n02.03.

2. https://www.minsal.cl/nuevocoronavirus-2019-ncov/informetecnico

3. Chen X, Liu Y, Gong Y, Guo X, Zuo M, Li J, et al.; Chinese Society of Anesthesiology, Chinese Association of Anesthesiologists. Perioperative Management of Patients Infected with the Novel
Coronavirus: Recommendation from the Joint Task Force of the Chinese Society of Anesthesiology and the Chinese Association of Anesthesiologists. Anesthesiology. 2020 Mar;2020:1 https://doi.org/10.1097/ ALN.0000000000003301 PMID:32195699

4. Horlocker TT, Vandermeuelen E, Kopp S, et al. Regional Anesthesia in the Patient Receiving Antithrombotic or Thrombolytic Therapy. American Society of Regional Anesthesia and Pain Medicine. Evidence-Based Guidelines. (Fourth Edition). Reg Anesth Pain Med 2018; 43: 263 309. https://doi.org/10.1097/ AAP.0000000000000763.

5. Tran K, Cimon K, Severn M, Pessoa-Silva CL, Conly J. Aerosol generating procedures and risk of transmission of acute respi- ratory infections to healthcare workers: a systematic review. PLoS One. 2012;7(4):e35797. https://doi.org/10.1371/journal. pone.0035797 PMID:22563403

6. Handbook of Covid-19 Prevention and Treatment. The first affiliated Hospital, Zhejiang University School of Medicine. Compiled According to Clinical Experience 2020.

7. Plà de Contigencia de Vall d'Hebron per fer front a I'epidèmia de SARS-CoV-2. Protocols HUVH 2020.

8. Recomendaciones prácticas para el manejo perioperatorio del paciente con sospecha o infección por coronavirus SARS-CoV-2 2020. www.sedar.es

9. Specialty guides for patient management during the coronavirus pandemic Clinical guide for the management of trauma 
and orthopaedic patients during the coronavirus pandemic. NHS 2020.

10. Peng PWH, Ho P-L, Hota SS. Outbreak of a new coronavirus: what anaesthetists should know. British Journal of Anaesthesia. Elsevier BV; 2020 Feb; http://dx.doi.org/10.1016/j. bja.2020.02.008

11. Rodriguez-Morales AJ, CardonaOspina JA, Gutiérrez-Ocampo E, Villamizar-Peña R, HolguinRivera Y, Escalera-Antezana JP, et al. Clinical, laboratory and imaging features of COVID-19: A systematic review and meta-analysis. Travel Medicine and Infectious Disease. Elsevier BV; 2020 Mar;101623. https://doi.org/10.1016/j. tmaid.2020.101623 PMID:32179124

12. Lippi G, Plebani M, Henry BM. Thrombocytopenia is associated with severe coronavirus disease 2019 (COVID-19) infections: A meta- analysis. Clin Chim Acta. 2020 Mar;506:145-8. https://doi. org/10.1016/j.cca.2020.03.022 PMID:32178975

13. Chen N, Zhou M, Dong X, Qu J, Gong F, Han Y, et al. Epidemiological and clinical characteristics of 99 cases of 2019 novel coronavirus pneumonia in Wuhan, China: a descriptive study. Lancet. 2020 Feb;395(10223):50713. https://doi.org/10.1016/ S0140-6736(20)30211-7 PMID:32007143 